\title{
Produção e qualidade de frutos de genótipos de amoreira-preta
}

\author{
Leonardo Oliboni do Amaral', Andrea De Rossi ${ }^{2}$, Ana Maria Alves de Souza Ribeiro', Hingrid Serafim², \\ Lucas de Ross Marchioretto'
' Embrapa Uva e Vinho - Estação Experimental de Fruticultura de Clima Temperado. Vacaria, RS, Brasil e Universidade do Estado de Santa Catarina - Centro de Ciências Agroveterinárias/CAV-UDESC - Programa de Pós-Graduação em Produção Vegetal. Lages, SC, Brasil.
E-mail: loamaral@ucs.br, anamaria.acre@gmail.com, lucasdeross@hotmail.com
2 Embrapa Uva e Vinho - Estação Experimental de Fruticultura de Clima Temperado. Vacaria, RS, Brasil.
E-mail: andrea.derossi@embrapa.br, hingridserafim@hotmail.com

Submetido em: 28 set. 2019. Aceito: 04 mar. 2020. DOI: http://dx.doi.org/10.21674/2448-0479.62.126-131

\section{Resumo}

Previamente ao lançamento de novas cultivares é importante a realização de estudos para observar o comportamento produtivo de plantas em diferentes condições edafoclimáticas. Este estudo teve como objetivo apresentar características de produção e qualidade físico-química de frutos de genótipos de amoreira-preta cultivados na região dos Campos de Cima da Serra do RS e indicar genótipos com potencial para serem lançados como cultivares ou seguirem no programa de melhoramento genético da cultura. O experimento foi conduzido em 2018, no município de Vacaria/RS, na Embrapa Uva e Vinho - Estação Experimental de Fruticultura de Clima Temperado. Foram avaliados os seguintes genótipos: Cultivares Tupy, Xingu e Xavante e seleções Black I I2, Black 139, Black I45, Black I8I, Black 198, Black 2 I 2, Black 2 I6, Black 287 e Black 288. Foram analisadas as seguintes variáveis: produção - produção por planta, produção por hectare e número de frutos por planta; pós-colheita - massa média, comprimento, diâmetro, relação comprimento/diâmetro dos frutos, sólidos solúveis, $\mathrm{pH}$ do suco e acidez total titulável. Observou-se que as seleções Black I45, Black I98 e Black 216 , devido à elevada produção de frutos, têm potencial para serem lançadas como novas cultivares, superando a cv. Tupy e igualando a produção da cv. Xingu. Já as seleções Black 287, Black 288 e Black 212 possuem aptidão para consumo in natura, devido às características químicas dos frutos.

Palavras Chave: Rubus. Melhoramento genético. Adaptação.

\section{Abstract}

\section{Production and fruit quality of blackberry genotypes}

Prior to the launch of new cultivars, it is important to carry out studies to observe the productive behavior of plants in each soil and climatic condition. The objective of this study was to present production and physical-chemical characteristics of fruits of blackberry genotypes cultivated in the Campos de Cima da Serra do Rio Grande do Sul and to indicate genotypes with potential to be released as cultivars or to follow in the breeding program of culture. The experiment was conducted in 2018, in the municipality of Vacaria/RS, in the Embrapa Uva e Vinho - Estação Experimental de Fruticultura de Clima Temperado. The following genotypes were evaluated: cultivars Tupy, Xingu and Xavante, and selections Black I I2, Black 139, Black I45, Black I8I, Black 198, Black 212, Black 216 and Black 288. The following variables were analyzed: production: Production per plant, production per hectare and number of fruits per plant; post-Harvest: average mass, length, diameter, soluble solids, juice $\mathrm{pH}$ and titratable total acidity. It was observed that the selections 
Black 145, Black 198 and Black 216, due to the high production of fruits, have the potential to be released as new cultivars, surpassing cv. Tupy and equaling the production of cv. Xingu. The Black 287, Black 288 and Black 212 selections are suitable for in natura consumption due to the chemical characteristics of the fruits.

Key-words: Rubus. Genetical enhancement. Adaptation.

\section{Introdução}

A cadeia produtiva da amora-preta no Brasil está passando por mudanças relacionadas à adoção de novas técnicas de produção, expansão das áreas de cultivo para regiões não tradicionais, busca de novas cultivares com características desejáveis e uma melhor organização da produção, logística e mercado, visando a maximização da rentabilidade da cultura.

O programa de melhoramento genético da amoreira-preta no Brasil, realizado pela Embrapa Clima Temperado em Pelotas/RS vem contribuindo através do lançamento de diversas cultivares, que visam atender as necessidades tanto de produtores quanto de consumidores, com relação às características de produção e qualidade de frutos.

Nesse contexto, a viabilidade do cultivo da amoreira-preta depende da adaptação das cultivares às condições edafoclimáticas de cada região, além disso, segundo Antunes e Raseira (2000), além da influência do clima, as características inerentes à cada genótipo podem influenciar no sistema produtivo e devem ser previamente testadas. Diante do exposto, o estudo do ciclo fenológico dos genótipos é de suma importância, não apenas sob o aspecto biológico, mas também como instrumento de planejamento do manejo dos pomares e da comercialização (HOJO et al., 2007). Já que a identificação de genótipos com variações na fenologia, para determinado local e especialmente com relação à época de colheita de frutos, tem implicação mercadológica relevante, pois colheitas mais precoces ou mais tardias permitem a comercialização em períodos de menor oferta de frutos (RAMOS; LEONEL, 2008).

Além da época de produção, o hábito de crescimento de plantas, vigor, ausência de espinhos e principalmente características de qualidade de frutos como a redução da acidez e aumento da vida de prateleira dos frutos, estão entre as prioridades dos programas de melhoramento genético da amoreira-preta no Brasil (RASEIRA; FRANZON, 20I2).

Dentro desse contexto, este estudo teve o objetivo de apresentar características de produção e qualidade físico-química de frutos de genótipos de amoreira-preta cultivados na região dos Campos de Cima da Serra do RS e indicar genótipos com potencial para serem lançados como cultivares ou seguirem no programa de melhoramento genético da cultura.

\section{Materiais e métodos}

O trabalho foi realizado na Estação Experimental de Fruticultura de Clima Temperado da Embrapa Uva e Vinho, localizada no município de Vacaria/RS, coordenadas geográficas $28^{\circ} 30^{\prime} 56,54^{\prime \prime}$ latitude sul e $50^{\circ} 53^{\prime}$ 08,50" longitude oeste. A altitude do local é de $967 \mathrm{~m}$ e clima classificado por Köppen como Cfb. As avaliações foram realizadas no ano de 2018, utilizando os seguintes genótipos oriundos do programa de melhoramento genético da amoreira-preta (Embrapa Clima Temperado): Black I I2, Black I39, Black I45, Black I8I (sem espinhos), Black 198, Black 2I2, Black 216, Black 287 e Black 288, as quais foram comparadas com as seguintes cultivares: Tupy, Xingu e Xavante (sem espinhos).

A área experimental foi implantada no ano de 2017 , em espaçamento de 0,5 m entre plantas e 4,0 m entre linhas, em delineamento experimental de blocos varietais casualizados, com 4 repetições, com os doze genótipos por bloco e 3 plantas úteis por parcela. $\bigcirc$ sistema de condução adotado foi o de espaldeira em "T", com altura de I m e travessa com dois fios de arame paralelos distanciados 0,5 m.

A colheita dos frutos foi realizada a cada três dias, quando os frutos estavam com coloração preta uniforme. Os frutos de cada parcela, correspondente a três plantas de cada genótipo, de cada bloco, eram acondicionados em bandejas de polietileno, identificadas e levadas ao laboratório, onde eram pesados em balança com precisão de Ig. Foi calculada a produção média por planta (kg planta-1), a produtividade estimada por hectare $(\mathrm{t} \mathrm{ha-1})$ e através da razão entre a produção de frutos por planta e a massa média dos frutos, foi calculado o número médio de frutos por planta de cada genótipo. 
Em pós-colheita foram avaliados os seguintes parâmetros físicos e químicos: massa média de frutos (g), comprimento e diâmetro equatorial de frutos $(\mathrm{mm})$, relação comprimento/diâmetro, sólidos solúveis totais ( ${ }^{\circ}$ Brix), potencial hidrogeniônico $(\mathrm{pH})$, acidez total titulável (ATT) - expressa em \% de ácido cítrico e relação SS/ATT (Ratio).

Os dados foram submetidos à análise de variância, e variáveis com efeito significativo foram submetidas ao teste de Scott-Knott a 5\% de probabilidade de erro. As análises foram realizadas utilizando-se o software estatístico SISVAR, versão 5.6 (FERREIRA, 2008).

\section{Resultados e discussão}

A produção por planta e a produtividade estimada por hectare são parâmetros diretamente relacionados e ambos variaram significativamente de acordo com os genótipos de amoreira-preta, testados. As seleções Black 216, Black 145, Black 198 e a cv. Xingu proporcionaram as maiores produtividades, alcançando médias superiores a 3,0 kg planta- ${ }^{-1}$, com destaque para a seleção Black 216 , a qual alcançou média de $3,80 \mathrm{~kg} \mathrm{planta}^{-1}$, conforme a Tabela I.

As seleções Black 145, Black 198 e a cv. Xingu já haviam demonstrado a característica de serem mais produtivas que a cv. Tupy em trabalho realizado por Vignolo et al (20I7), onde esses genótipos apresentaram produção de cerca de $1 \mathrm{I}, 0$ ton ha ${ }^{-1}$, praticamente o dobro da produção obtida com a cv. Tupy, na condição de Pelotas/RS, em pomar com 4 anos de implantação. As seleções Black I I2, Black I39, Black 2I 2, Black 287 e Black 288 foram semelhantes à cv. Tupy, sendo que esta produziu $2,69 \mathrm{~kg}$ planta $^{-1}$ (Tabela I). Já a seleção Black I8I e a cv. Xavante (ambas sem espinhos) foram as menos produtivas, alcançando médias de I,0 kg planta $^{-1}$ e I, $16 \mathrm{~kg}_{\text {planta }}{ }^{-1}$, respectivamente.

Os índices de produtividade observados foram superiores aos relatados por Santos, Raseira e Madail (1997), os quais obtiveram para a cv. Tupy, em região de clima temperado, produtividade de 5,0 ton ha-1 no primeiro ano.

No presente experimento foram obtidas 13,49 ton ha ${ }^{-1}$ já no primeiro ano de cultivo para a cv. Tupy e produtividades iguais ou superiores a esta, para vários genótipos avaliados (Tabela I). Diversos fatores podem ter contribuído para esse resultado ter sido distinto ao relatado na literatura, onde pode ser citado o satisfatório acúmulo de frio hibernal para superação da dormência das gemas, manejo da adubação, uniformidade na condução de plantas, uso de irrigação na área experimental, somando-se à reduzida perda de frutos em vista das colheitas periódicas e no ponto correto de maturação de frutos.

Os frutos com maior massa foram oriundos da cv. Tupy e das seleções Black I I2, Black I45, Black 2 I 2 e Black 216, portanto, não houve genótipos com frutos de maior massa quando comparados à cv. Tupy (Tabela I). Souza (20l3) encontrou valores de massa média para frutos da seleção Black I45 de II g, valor acima do presente experimento. Em contrapartida, os frutos de menor massa foram oriundos das seleções Black I8I e Black 287. A seleção Black 287 foi a que produziu o maior número de frutos por planta, no entanto, devido ao reduzido tamanho e consequentemente baixa massa média de frutos, a produtividade foi afetada de forma negativa.

Os resultados de massa média de frutos observados para as cvs. Tupy e Xavante estão de acordo com o relatado por Antunes e Raseira (2004). Já Brugnara (2016), conduzindo experimento em Santa Catarina, com cinco cultivares de amoreira-preta, observou massa média de 6,58 g para a cv. Tupy e 4,56 g para a cv. Xavante, estando abaixo do encontrado no presente trabalho, onde igualmente foi obtida maior massa para a cv. Tupy em comparação à 'Xavante', no entanto com médias de 8,03 g e $5,86 \mathrm{~g}$, respectivamente.

Tais resultados demonstram que a região produtora, os fatores climáticos de cada ciclo produtivo e o manejo adotado podem alterar as características de produção e qualidade de frutos dos genótipos. Entre as condições climáticas, o índice pluviométrico é um fator que pode influenciar na massa fresca dos frutos, pois com maior quantidade de água disponível no solo, maior é a absorção pela planta e consequentemente maior a turgescência de células, aumentando a massa média. Neste sentido, salienta-se que na área experimental foi utilizada irrigação por gotejamento nos períodos com baixa pluviosidade.

Observou-se diferença significativa entre os genótipos para o diâmetro e comprimento dos frutos. Foi 
observada menor variação no diâmetro (cerca de 3,0 mm entre o menor e o maior diâmetro) e variação mais acentuada (cerca de 10,0 mm) no comprimento dos frutos entre os genótipos (Tabela I). A maior média de diâmetro foi observada para a seleção Black 216 , a qual não diferiu estatisticamente das médias encontradas para as seleções Black II2, Black 139, Black 145 e cv. Tupy. Os menores diâmetros foram obtidos para as seleções Black I8I e Black 287.

Analisando-se o comprimento de frutos, nota-se que as menores médias foram observadas para as seleções Black I8I e Black 287 (Tabela I). Já a seleção Black 212 apresentou a maior média, com 31,78 mm de altura, seguida pela seleção Black 145, cv. Tupy e Black 216 . A diferença entre o diâmetro $(20,74 \mathrm{~mm}) \mathrm{e}$ a altura $(31,78 \mathrm{~mm})$ da seleção Black 212 se refletiu na maior média de relação comprimento/diâmetro dos frutos, o que confere a esta seleção o formato de fruto alongado/cilíndrico. Tal fator é também perceptível para as seleções Black I45, Black 198 e Black 288, contudo, em menor grau.

Antunes e Raseira (2018) observaram resultados similares quanto aos frutos da seleção Black 212 , encontrando médias de comprimento e diâmetro de $30 \mathrm{~mm}$ e $19 \mathrm{~mm}$, respectivamente. Os mesmos autores destacam o tamanho e formato de fruto alongado, conferindo ótima aparência aos frutos desta seleção. As menores relações de comprimento/diâmetro e, consequentemente, o formato de fruto mais esférico, foram observadas para a cv. Xavante e seleções Black I39, Black I8I e Black 287 (Tabela I).

Tabela I - Produção por planta, produtividade estimada, número de frutos por planta, massa média, diâmetro, comprimento e relação comprimento/diâmetro de frutos de genótipos de amoreira-preta. Vacaria - RS, 2019.

\begin{tabular}{cccccccc}
\hline Genótipos & $\begin{array}{c}\text { Produção } \\
\text { por planta } \\
(\mathrm{kg})\end{array}$ & $\begin{array}{c}\text { Produtividade } \\
\text { estimada } \\
(\mathrm{t} \mathrm{ha})^{-1}\end{array}$ & $\begin{array}{c}\text { Número de } \\
\text { frutos por } \\
\text { planta }\end{array}$ & $\begin{array}{c}\text { Massa média } \\
\text { de frutos } \\
(\mathrm{g})\end{array}$ & $\begin{array}{c}\text { Diâmetro } \\
\text { de frutos } \\
(\mathrm{mm})\end{array}$ & $\begin{array}{c}\text { Comprimento } \\
\text { de frutos } \\
(\mathrm{mm})\end{array}$ & $\begin{array}{c}\text { Relação } \\
\text { comprimento/diâmetro } \\
\text { de frutos }\end{array}$ \\
\hline Tupy & $2,69 \mathrm{~b}$ & $13,49 \mathrm{~b}$ & $338,06 \mathrm{c}$ & $8,03 \mathrm{a}$ & $22,02 \mathrm{a}$ & $28,50 \mathrm{~b}$ & $1,29 \mathrm{~b}$ \\
Xingu & $3,25 \mathrm{a}$ & $16,29 \mathrm{a}$ & $502,61 \mathrm{~b}$ & $6,49 \mathrm{~b}$ & $20,66 \mathrm{~b}$ & $25,74 \mathrm{c}$ & $1,24 \mathrm{c}$ \\
Xavante & $1,16 \mathrm{c}$ & $5,82 \mathrm{c}$ & $198,17 \mathrm{~d}$ & $5,86 \mathrm{~b}$ & $20,47 \mathrm{~b}$ & $23,82 \mathrm{~d}$ & $1,16 \mathrm{~d}$ \\
Black 112 & $2,41 \mathrm{~b}$ & $12,05 \mathrm{~b}$ & $310,03 \mathrm{c}$ & $7,8 \mathrm{a}$ & $21,70 \mathrm{a}$ & $26,26 \mathrm{c}$ & $1,21 \mathrm{c}$ \\
Black 139 & $2,39 \mathrm{~b}$ & $11,98 \mathrm{~b}$ & $345,42 \mathrm{c}$ & $6,90 \mathrm{~b}$ & $21,85 \mathrm{a}$ & $25,46 \mathrm{c}$ & $1,16 \mathrm{~d}$ \\
Black 145 & $3,44 \mathrm{a}$ & $17,20 \mathrm{a}$ & $394,74 \mathrm{~b}$ & $8,70 \mathrm{a}$ & $21,33 \mathrm{a}$ & $29,08 \mathrm{~b}$ & $1,36 \mathrm{~b}$ \\
Black 18I & $1,00 \mathrm{c}$ & $5,44 \mathrm{c}$ & $210,43 \mathrm{~d}$ & $4,83 \mathrm{c}$ & $19,5 \mathrm{c}$ & $21,79 \mathrm{e}$ & $1,12 \mathrm{~d}$ \\
Black 198 & $3,03 \mathrm{a}$ & $15,16 \mathrm{a}$ & $464,14 \mathrm{~b}$ & $6,55 \mathrm{~b}$ & $20,76 \mathrm{~b}$ & $26,61 \mathrm{c}$ & $1,28 \mathrm{~b}$ \\
Black 212 & $2,22 \mathrm{~b}$ & $11,14 \mathrm{~b}$ & $254,03 \mathrm{~d}$ & $8,73 \mathrm{a}$ & $20,74 \mathrm{~b}$ & $31,78 \mathrm{a}$ & $1,53 \mathrm{a}$ \\
Black 216 & $3,80 \mathrm{a}$ & $19,02 \mathrm{a}$ & $457,74 \mathrm{~b}$ & $8,38 \mathrm{a}$ & $22,89 \mathrm{a}$ & $28,24 \mathrm{~b}$ & $1,23 \mathrm{c}$ \\
Black 287 & $2,66 \mathrm{~b}$ & $13,30 \mathrm{~b}$ & $592,17 \mathrm{a}$ & $4,52 \mathrm{c}$ & $19,01 \mathrm{c}$ & $21,46 \mathrm{e}$ & $1,13 \mathrm{~d}$ \\
Black 288 & $2,88 \mathrm{~b}$ & $14,4 \mathrm{~b}$ & $454,32 \mathrm{~b}$ & $6,37 \mathrm{~b}$ & $20,46 \mathrm{~b}$ & $26,66 \mathrm{c}$ & $1,31 \mathrm{~b}$ \\
\hline CV (\%) & 18,25 & 18,25 & 16,69 & 8,2 & 4,21 & 3,7 & 4,17 \\
\hline
\end{tabular}

Médias seguidas de mesma letra, na coluna, não diferem estatisticamente entre si pelo teste de Scott-Knott $(P<0,05)$. ${ }^{A}$ produtividade estimada foi calculada com base em uma densidade de 5000 plantas ha $^{-1}$.

Fonte: Autores, 2019.

A avaliação das características químicas de frutos de amoreira-preta é de suma importância, tendo em vista que podem ocorrer variações significativas nos atributos, entre os genótipos. Em consequência, características de produtividade e qualidade de frutos, definem as melhores aptidões: mercado in natura ou para industrialização, na forma dos mais diversos produtos.

O teor de sólidos solúveis (SS) é um importante indicador de grau de maturação de alguns frutos, o qual se baseia na quantificação de substâncias que estão dissolvidas no suco, sobretudo os açúcares. Observou-se no experimento, uma variação na faixa de $8,15^{\circ}$ Brix a $9,75^{\circ}$ Brix, sendo que os maiores valores foram observados nos frutos dos genótipos sem espinhos: cv. Xavante $\left(9,75^{\circ}\right.$ Brix $)$ e seleção Black I8I $\left(9,51^{\circ}\right.$ Brix) (Tabela 2). As seleções Black 212, Black 216 e Black 288 apresentaram valores em torno de 9,0 ${ }^{\circ}$ Brix. Em seguida, situaram-se a cv. Xingu e as seleções Black II2, Black 198 e Black 287 com aproximadamente $8,5^{\circ} \mathrm{Brix}$. Já com sólidos solúveis na faixa de similaridade à $\mathrm{cv}$. Tupy, que foi de $8,21^{\circ} \mathrm{Brix}$, se posicionaram as seleções Black 139 e Black 145. 
As médias de sólidos solúveis encontradas para cv. Tupy estão de acordo com Antunes e Raseira (2004), o qual indica uma variação entre 8 e $9^{\circ}$ Brix para esta cultivar. De acordo com Hassimoto et al (2008), os valores de sólidos solúveis das principais cultivares de amoreiras-pretas cultivadas no Brasil situam-se na faixa

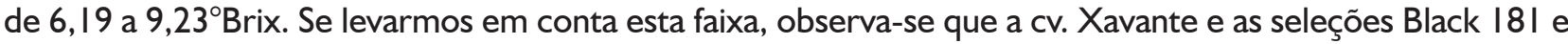
Black 216 apresentaram resultados superiores (Tabela 2).

Com relação ao $\mathrm{pH}$ do suco obtido dos frutos das cultivares e seleções, houve pouca variação, ficando entre 2,36 e 2,6I (Tabela 2). A cv. Xingu e as seleções Black 198 e Black 287 diferiram estatisticamente das demais, com $\mathrm{pH}$ levemente mais alto.

Já a acidez total titulável (ATT), expressa em \% de ácido cítrico, diferiu de forma mais acentuada entre os genótipos. A seleção Black 287 apresentou ATT mais baixa que todos os demais genótipos, com $0,95 \%$ (Tabela 2). A baixa acidez da seleção Black 287 , associada ao índice de $8,49^{\circ}$ Brix de SS refletiu na maior média de relação entre SS/ATT (ratio). De acordo com Souza (20l3), o ratio pode ser definido como a relação correspondente entre o conteúdo de acidez e de açúcares presentes nos frutos, sendo um parâmetro apropriado para medir a percepção do sabor pelo consumidor.

Para a cv. Tupy e seleções Black 198 e Black 216, foram observadas porcentagens intermediárias de acidez, enquanto os maiores valores foram observados para as cultivares Xingu, Xavante e seleções Black II 2, Black 139, Black I45 e Black I8I (Tabela 2). Os valores de ATT encontrados para as cvs. Tupy e Xavante estão de acordo com os relatados por Brugnara (2016).

Excluindo-se a seleção Black 287, observa-se que a seleção Black 2 I 2 destacou-se das demais com baixa acidez (I,II\%), e da mesma forma, elevando o valor de relação SS/ATT para 8, I I (Tabela 2). Os resultados obtidos para a seleção Black 212 nesse estudo estão de acordo com o encontrado por Antunes e Raseira (20l8), os quais destacam que frutos dessa seleção não são tão doces como prefere a maioria dos consumidores, mas no mínimo são comparáveis à cv. Tupy. No entanto, apresentam melhor relação SS/ATT, o que acaba conferindo melhor sabor. Observa-se que a seleção Black 288 também possui boa relação SS/ATT, demonstrando juntamente com as seleções Black 287 e Black 212, aptidão para o consumo in natura.

Pereira et al (2014) e Antunes e Raseira (2004) relatam que a cultivar Xavante caracteriza-se pela elevada produtividade, mas os frutos possuem elevada acidez e amargor ao paladar, sendo assim destinados sobretudo à industrialização. De fato, observou-se que os frutos dos genótipos sem presença de espinhos (Black I8I e cv. Xavante) apresentaram os maiores conteúdos de sólidos solúveis, no entanto, também os maiores valores de acidez. Portanto, pode-se inferir que o amargor característico da cv. Xavante se deve à elevada acidez, que sobressai em detrimento aos sólidos solúveis.

Tabela 2 - pH, teor de sólidos solúveis, acidez total titulável e relação SS/ATT de genótipos de amoreira-preta. Vacaria - RS, 2019.

\begin{tabular}{|c|c|c|c|c|}
\hline Genótipos & $\mathrm{pH}$ & Teor de sólidos solúveis ( ${ }^{\circ}$ Brix) & Acidez total titulável (\%) & Relação SS/ATT \\
\hline Tupy & $2,49 \mathrm{~b}$ & $8,21 \mathrm{~d}$ & $\mathrm{I}, 23 \mathrm{~b}$ & $6,71 \mathrm{~d}$ \\
\hline BRS Xingu & $2,56 \mathrm{a}$ & $8,72 c$ & $\mathrm{I}, 50 \mathrm{a}$ & 5,84 e \\
\hline Xavante & $2,36 \mathrm{~b}$ & $9,75 \mathrm{a}$ & $\mathrm{I}, 5 \mathrm{I} \mathrm{a}$ & $6,48 d$ \\
\hline Black 112 & $2,46 \mathrm{~b}$ & $8,42 \mathrm{c}$ & $\mathrm{I}, 48 \mathrm{a}$ & $5,69 \mathrm{e}$ \\
\hline Black 139 & $2,38 \mathrm{~b}$ & $8,15 \mathrm{~d}$ & $\mathrm{I}, 55 \mathrm{a}$ & 5,26 e \\
\hline Black 145 & $2,43 \mathrm{~b}$ & $8,16 d$ & $\mathrm{I}, 47 \mathrm{a}$ & 5,55 e \\
\hline Black |8| & $2,38 \mathrm{~b}$ & $9,51 \mathrm{a}$ & $\mathrm{I}, 46 \mathrm{a}$ & $6,52 \mathrm{~d}$ \\
\hline Black 198 & $2,58 \mathrm{a}$ & $8,65 c$ & $\mathrm{I}, 27 \mathrm{~b}$ & $6,84 \mathrm{~d}$ \\
\hline Black 212 & $2,43 \mathrm{~b}$ & $9,01 \mathrm{~b}$ & $\mathrm{I}, \mathrm{II} \mathrm{d}$ & $8,11 \mathrm{~b}$ \\
\hline Black 216 & $2,4 I b$ & $9,26 \mathrm{~b}$ & $\mathrm{I}, 27 \mathrm{~b}$ & $7,31 \mathrm{c}$ \\
\hline Black 287 & $2,61 \mathrm{a}$ & $8,49 c$ & 0,95 e & $8,92 \mathrm{a}$ \\
\hline Black 288 & $2,43 \mathrm{~b}$ & $9,12 b$ & $1,18 \mathrm{c}$ & $7,73 \mathrm{~b}$ \\
\hline CV (\%) & 2,81 & 3,30 & 3,40 & 5,29 \\
\hline
\end{tabular}

Médias seguidas de mesma letra, na coluna, não diferem estatisticamente entre si pelo teste de Scott-Knott $(P<0,05)$. Fonte: Autores, 2019. 


\section{Conclusões}

As seleções Black 145, Black 198 e Black 216, devido à elevada produção de frutos, têm potencial para serem lançadas como novas cultivares, sobretudo visando a industrialização.

As seleções Black 287, Black 288 e Black 212 possuem aptidão para consumo in natura, devido às características químicas dos frutos.

\section{Agradecimentos}

À Estação Experimental de Fruticultura de Clima Temperado da Embrapa Uva e Vinho, pela disponibilização da área experimental e dos laboratórios e à Coordenação de Aperfeiçoamento de Pessoal de Nível Superior (CAPES) pelo fomento da bolsa de pesquisa.

\section{Referências}

ANTUNES, L. E. C.; RASEIRA, M. C. B. Introdução Geral. In: (Orgs.). Aspectos técnicos da cultura da amoreira-preta. Pelotas: Embrapa Clima Temperado, 2004, cap. I, p. II-I2.

ANTUNES, L. E. C.; RASEIRA, M. C. B. Floração e períodos de maturação das variedades de amoreira-preta no Brasil. Journal American Pomological Society, v. 54, n. 4, p. 164-168, 2000. Disponível em: http://www.pubhort.org/ aps/54/v54_n4_a31.htm. Acesso em: 20 jun. 2018.

ANTUNES, L. E. C; RASEIRA, M. C. B. Fruticultura: Cultivar de amora-preta BRS Cainguá e técnicas de cultivo do mirtilo. In: WOLFF, L. F; MEDEIROS, C. A. B. (Eds.). Alternativas para a diversificação da agricultura familiar de base ecológica. Pelotas: Embrapa Clima Temperado, 2018.63 p. Disponível em: https://www.infoteca.cnptia.embrapa. br/infoteca/bitstream/doc/l 101 1084/l/LuisEduardoCorreaDOCUMENTOS467.indd.pdf. Acesso em: 02 jan. 2019.

BRUGNARA, E. C. Produção, época de colheita e qualidade de cinco variedades de amoreira-preta em Chapecó, SC. Agropecuária Catarinense, Florianópolis, v. 29, n. 3, p. 7I-75, set./dez. 2016.

FERREIRA, D.F. SISVAR. Um programa para análises e ensino de estatística. Revista Symposium, Lavras, v. 6 , p. 36-4I, 2008.

HASSIMOTO, N. M. A. et al. Physico-chemical characterization and bioactive compounds of blackberry fruits (Rubus sp.) grown in Brazil. Ciência e Tecnolgia de Alimentos, Campinas, v. 28, n. 3, p. 702-708, 2008.

HOJO, R. H. et al. Caracterização fenológica da goiabeira 'Pedro Sato' sob diferentes épocas de poda. Revista Brasileira de Fruticultura, Cruz das Almas, v. 29, n. I, p. 20-24, 2007.

PEREIRA, I. S. et al. Informações técnicas de cultivares de amoreira-preta. Pelotas: Embrapa Clima Temperado, 2014.

RAMOS, D. P.; LEONEL, S. Características dos frutos de cultivares de pessegueiros e nectarineira, com potencial de cultivo em Botucatu, SP. Bioscience Journal, v. 24, p. 10-18, 2008.

RASEIRA, M. C. B; FRANZON, R. C. Melhoramento genético e cultivares de amoreira-preta e mirtilo. Informe Agropecuário, Belo Horizonte, v.33, n.268, p.I I-20, 2012.

SANTOS, A. M.; RASEIRA, M. C. B.; MADAIL, J. C. M. Amora-preta. Brasília: Embrapa Clima Temperado, 1997, 2. ed.

SOUZA, A. V. Pós-colheita e armazenamento de amora-preta 'Tupy'. 2013. Tese de doutorado - Programa de Pós-Graduação em Agronomia, Universidade Estadual Paulista, Botucatu, 2013.

VIGNOLO, G. K. et al. Fenologia e produção de seleções e cultivares de amoreira-preta em Pelotas-RS. In: SEMINÁRIO BRASILEIRO SOBRE PEQUENAS FRUTAS, 2017, Vacaria. Anais... Vacaria, 2017. Disponível em: http://conferencia. uergs.edu.br/index.php/sbpf/sbpf/paper/view/2050 Acesso em: 18 dez. 2017. 